\title{
TRAITEMENT DE SURFACE : UN OUTIL POUR ÉVALUER LES ALTERNATIVES DE TRAITEMENT DES REJETS
}

\author{
B. Debray, M. Leduc, J. Bourgois* \\ École des mines de Saint-Étienne
}

\begin{abstract}
L'industrie du traitement de surface produit en grande quantité des effluents toxiques dont elle doit impérativement réduire la toxicité avant rejet dans le milieu naturel. Se pose alors le problème du choix d'une solution de traitement adaptée et économiquement acceptable. En raison d'un manque d'information sur les techniques disponibles les solutions retenues sont souvent inadaptées et font rarement appel à la valorisation qui a pourtant été fixée comme priorité. Pour tenter de remédier à ce problème, nous développons un outil informatique d'aide à la décision pour le choix de solutions de traitement. Un exemple illustre les fonctionnalités actuelles du système et les compléments que nous tentons d'y apporter en modélisant la détoxication des effluents par voie physico-chimique (oxydoréduction, neutralisation, précipitation).
\end{abstract}

The electroplating and surface finishing industry produces heavy metal bearing waste waters that must be treated before disposal. The choice of an appropriate treatment technique depends on good knowledge of the available means and of the parameters that determine their aplicability. In this context we are developing a decision data system to help the choice of treatment technologies oriented towards waste revaluation.

\section{INTRODUCTION}

Les entreprises de traitement de surface font actuellement face à un double défi : survivre dans un contexte économique difficile et mettre en place une politique environnementale permettant d'atteindre les normes de rejets imposées par les pouvoirs publics. De ces deux préoccupations contradictoires naissent des conflits que les petites entreprises ont souvent du mal à résoudre. En particulier, bien que conscientes de la nécessité de trouver des solutions pour leurs rejets, elles sont peu enclines à investir dans des infrastructures lourdes qui pourraient se révéler à terme inopérantes car mal adaptées à leurs besoins. Devant la difficulté d'accès à l'information concernant les procédés de traitement et la complexité des raisonnements qui interviennent dans le choix d'une solution, beaucoup d'industriels se découragent.

C'est pourquoi nous avons entrepris de développer un outil d'aide à la décision pour le choix de procédés de valorisation des effluents de traitement de surface (TS-Clean). L'objectif de cet outil n'est pas de remplacer une étude de faisabilité, mais de sensibiliser les industriels du traitement de surface aux possibilités qu'offre la valorisation, en faisant apparaître les différentes solutions qu'ils peuvent mettre en œuvre, en tentant d'évaluer l'intérêt de chaque solution par rapport aux autres. Dans cet article nous décrirons succinctement les objectifs et la structure de cet outil puis nous insisterons sur les calculs physico-chimiques destinés à l'évaluation des solutions classiques de traitement par détoxication.

\section{CONTRAINTES ET MOTIVATIONS POUR LE CHOIX D'UNE SOLUTION DE TRAITEMENT}

Les rejets de l'industrie du traitement de surface sont soumis à l'arrêté du 26 septembre 1985' qui fixe les seuils de concentration admise dans les effluents. Cet arrêté définit aussi la quantité d'eau maximale utilisable pour une surface de pièce traitée. L'objectif d'un traiteur de surface sera donc à la fois de respecter les normes de rejet et de diminuer sa consommation d'eau. L'arrêté de 85 fixe donc les objectifs minima de l'industriel. La loi du 13 juillet $1992^{2}$ fait de la réduction à la source ou à défaut la valorisation des déchets des priorités. En théorie, ces deux pratiques devraient conduire à des économies et sont donc compatibles avec les contraintes financières des industriels. Elles vont aussi dans le sens des économies d'eau demandées par l'arrêté de 1985. Malheureusement, les techniques de valorisation sont mal connues des industriels ; nécessitant dans certains cas un investissement très important, elles souffrent d'une mauvaise réputation amplifiée par des problèmes de fiabilité et de maîtrise. En revanche, les techniques classiques de détoxication sont souvent bien connues, bien maîtrisées pour des effluents simples et relativement faciles d'emploi. Mais elles ne répondent pas aux objectifs de valorisation fixés par la loi du I 3 juillet 1992 et leur simplicité cache parfois une inaptitude au traitement d'effluents complexes. Enfin, elles entraînent la plupart du temps des frais de fonctionnement élevés. Pour pouvoir comparer les avantages et inconvénients des différentes solutions, il est indispensable de les connaître 
et de savoir en apprécier la pertinence. C'est un des objectifs fixés à TS-Clean.

Une solution de traitement devra être évaluée en fonction de sa capacité à réduire la pollution jusqu'à des concentrations acceptables, son potentiel de valorisation et, enfin, son coût. La démarche qui conduit à l'évaluation d'une solution passe par : - un diagnostic de la pollution à éliminer,

- une identification des procédés théoriquement applicables et de leurs objectifs,

- la détection d'incompatibilités liées à la composition particulière de l'effluent,

- l'évaluation de l'efficacité des solutions envisagées en fonction des contraintes de rejet,

- le calcul des coûts d'investissement et de fonctionnement ainsi que l'identification des économies réalisées grâce à une solution de type valorisation.

\section{DESCRIPTION DE L'OUTIL TS-CLEAN}

TS-Clean a déjà fait l'objet d'une description dans la littérature $^{3}$ dont nous rappellerons ici les points essentiels en insistant sur les compléments que nous cherchons à y apporter. L'enchaînement des écrans (figure I) du mode utilisateur donne par ailleurs une idée assez précise des fonctions disponibles dans TS-Clean.

\section{Phase de diagnostic}

Actuellement le diagnostic fait intervenir deux paramètres : la fonction de traitement de surface mise en œuvre et la composition des effluents. La connaissance de la fonction de traitement de surface permet d'identifier les principaux composants de l'effluent et la catégorie de déchet à laquelle il appartient. On peut ainsi proposer à l'utilisateur une composition type correspondant à cette catégorie de déchets, évitant ainsi les omissions ou les aberrations. La fonction de traitement de surface est aussi essentielle dans la phase d'identification des solutions de valorisation applicables.

Lorsque la composition type de l'effluent a été déterminée pour chaque poste (bain de traitement, bain de rinçage), l'utilisateur a la possibilité de préciser les concentrations en différents éléments. Ces concentrations sont comparées avec les normes de rejet définies dans l'arrêté du 26 septembre 1985. Elles servent de base au calcul des quantités de réactifs à mettre en œurre pour une détoxication physico-chimique classique.

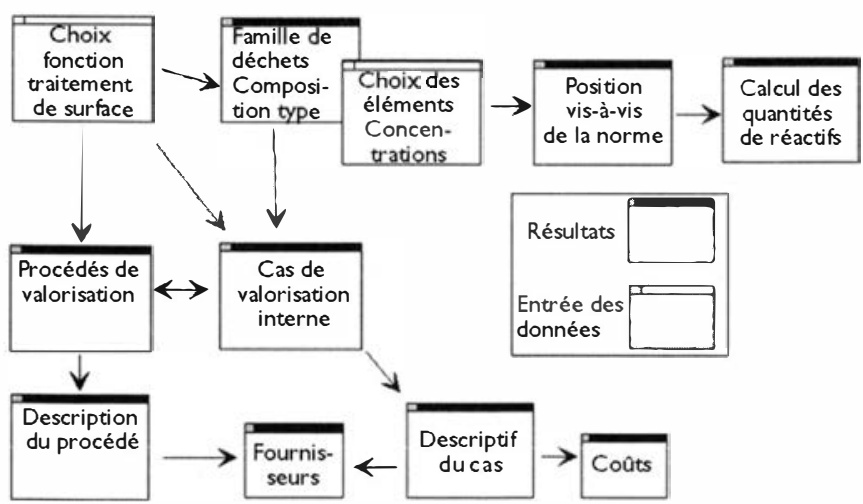

Figure I : Enchaînement des écrans dans TS-Clean

\section{IDENTIFICATION DES PROCÉDÉS APPLICABLES}

Il faut distinguer le cas de la valorisation des effluents et celui d'un traitement classique de détoxication. Actuellement, l'identification d'une solution de valorisation passe par une base de cas réels. Lorsque la phase de diagnostic est terminée, la base est consultée et les solutions les plus proches sont proposées à l'utilisateur. La comparaison porte sur la fonction de traitement de surface concernée et sur les polluants présents dans l'effluent. L'utilisateur a alors accès à la liste des procédés de valorisation envisageable ou bien à la liste des cas identifiés. Dans le premier cas il peut consulter la description du procédé et des informations théoriques alors que dans le deuxième cas il peut avoir les détails concernant les coûts de mise en œuvre d'une installation réelle.

Une détoxication classique par le biais d'un traitement physico-chimique des effluents fait actuellement l'objet, dans TSClean, d'un calcul sommaire des quantités de réactifs nécessaires à la précipitation des métaux. Nous travaillons actuellement à la généralisation de ce module afin d'obtenir une information plus fiable et plus pertinente. Nous poursuivons trois objectifs : - identifier de manière explicite et complète les opérations à enchaîner pour le traitement des effluents. Cela implique de prendre en compte une plus grande variété de réactifs et d'opérations unitaires ;

- détecter les problèmes qui peuvent apparaître en raison notamment de la présence d'éléments incompatibles dans les effluents. Ce peut être des problèmes de domaines de précipitation différents pour des métaux ou de complexants qui modifient le comportement des éléments en présence ; - enfin, intégrer un véritable calcul des quantités de boues générées.

Ces améliorations devraient permettre de mieux évaluer une solution classique de traitement de type précipitation filtration, tant sur le plan du coût avec la quantité de réactifs nécessaires et la quantité de boue formée, que sur le plan de l'efficacité. On pourra ainsi mieux mettre en évidence l'apport de solutions de type valorisation qui peuvent intervenir en remplacement ou en complément d'une solution classique.

\section{EXEMPLE D'APPLICATION}

L'exemple suivant permettra de mieux cerner le fonctionnement de TS-Clean et l'apport des modifications envisagées. Nous nous plaçons dans le contexte d'un atelier de traitement de surface pratiquant un cadmiage alcalin et un chromage dur. Les effluents produits sont donc du type :

- alcalins cyanurés et cadmiés à l'issu de l'opération de cadmiage ; - chromiques en sortie de la chaîne de chromage.

Dans un contexte classique le traitement des effluents est réalisé dans une même station de détoxication décrite schématiquement en figure 2 .

Le raisonnement en vue de l'évaluation de ce procédé de traitement comporte les étapes suivantes :

- identification des espèces en solution : ceci conduit à différencier dans le cas présent les ions $\mathrm{CrO}_{4}{ }^{2-}, \mathrm{HCrO}_{4}{ }^{\circ}$ et 


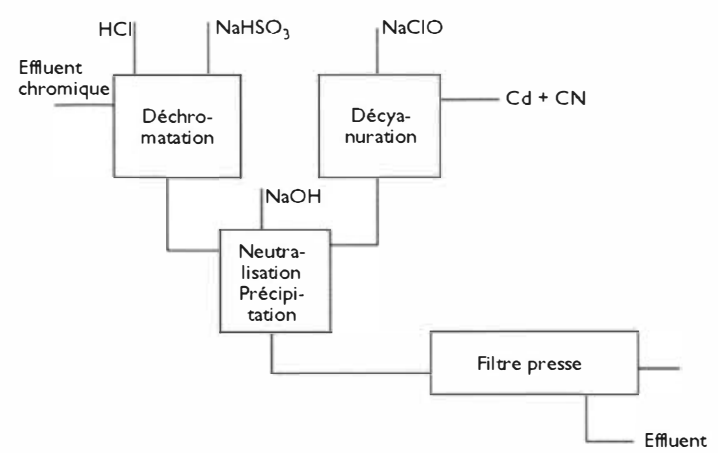

Figure 2 : Schéma d'une station de détoxication pour des effluents chromiques et des effluents cadmiés et cyanurés

$\mathrm{Cr}_{2} \mathrm{O}_{7}{ }^{2-}$ en fonction du $\mathrm{pH}$ et du titre en $\mathrm{Cr}$. La nature de l'espèce présente intervient dans le calcul de la quantité d'acide à ajouter pour ajuster le $\mathrm{pH}$ de la solution chromique à la valeur idéale pour la réduction des ions chromiques ; - détermination des étapes du traitement : décyanuration suivie d'une précipitation pour l'effluent cadmié cyanuré, acidification, puis réduction du chrome VI en chrome III et, enfin, précipitation pour l'effluent chromique.

- identification des conflits : sur la figure 3 sont représentées les courbes de solubilité du cadmium et du chromell en fonction du $\mathrm{pH}$. Ces courbes, modélisées sous forme de segments de droite et introduites au niveau de l'outil informatique permettent de détecter les incompatibilités. Ici, la zone de coprécipitation du chrome et du cadmium est très réduite, ce qui conduira très probablement à des rejets accidentels en chrome ou en cadmium dans un contexte industriel. - calcul des quantités de réactifs à utiliser et des quantités de boue générée. Ici le calcul permet simplement d'évaluer le coût approximatif d'un traitement classique à condition de connaitre les prix des réactifs utilisés (soude, bisulfite de sodium, hypochlorite de sodium ou acide persulfurique). En revanche, le calcul des quantités de boues générées nécessite de pouvoir évaluer la densité des boues obtenues après pressage.

\section{SOLUTIONS ALTERNATIVES}

Le raisonnement exposé ci-dessus a mis en évidence des dif-

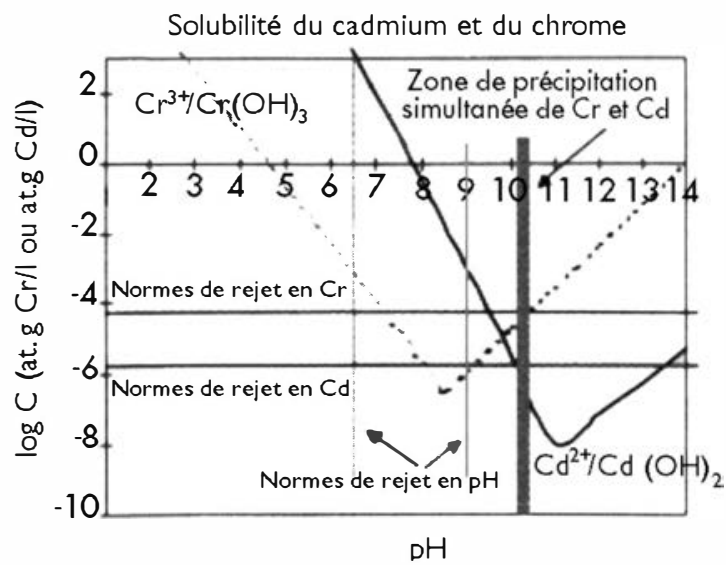

Figure 3 : Solubilité du cadmium et du chrome en fonction du pH d'après M. Pourbaix 4 . Les normes de rejet correspondent à celles exigées par l'arrêté du 26 septembre 1985 ficultés dans l'élimination simultanée du chrome et du cadmium par une solution physico-chimique classique. Pour contourner ces difficultés, il est possible de modifier la chaîne de traitement en ajoutant des étapes intermédiaires de précipitation et de filtration (en fin de décyanuration, par exemple) ou bien faire intervenir des solutions de valorisation qui limitent les besoins en termes d'épuration des effluents.

Pour les fonctions concernées, TS-Clean fournit plusieurs techniques associées à des exemples de réalisations relevés dans la littérature :

- l'évaporation ${ }^{5}$, qui permet, par concentration de l'effluent issu d'un rinçage statique, de régénérer le bain de traitement tout en récupérant l'eau épurée pour les rinçages. Cette solution est proposée seule pour le cadmiage et en association avec un traitement par résine pour le chromage ;

- l'électrolyse ${ }^{6}$ sur les bains de rinçage de l'effluent cadmié, qui permet de récupérer le cadmium et de réduire la concentration dans le bain de rinçage statique et, par conséquent la teneur en cadmium dans les rinçages courants ;

- l'électro-électrodialyse ${ }^{7}$ pour la régénération du bain de chromage et l'épuration du bain de rinçage statique.

Ces trois solutions permettent de limiter la teneur en chrome et cadmium dans les rejets, ce qui diminue les risques de pollution et la quantité de réactifs à utiliser lors de la détoxication. En associant ces dispositifs à des échangeurs ioniques, il est possible de se passer complètement de l'installation de traitement classique. Sur le plan financier, les exemples fournis par TS-Clean montrent des gains appréciables si l'on tient compte de l'économie de réactif réalisée. Nous n'avons cependant pas introduit dans l'outil de calcul rigoureux des coûts et une généralisation des exemples peut se révéler abusive. En revanche, la sécurité qu'apportent les solutions envisagées par rapport à la délicate précipitation du chrome et du cadmium doit être prise en compte dans le choix définitif d'une solution de traitement.

\section{CONCLUSIONS}

L'exemple d'un atelier pratiquant en même temps du chromage dur et du cadmiage a permis de montrer en quoi une meilleure modélisation du traitement classique de détoxication peut permettre de mettre en évidence des difficultés potentielles liées à la composition des effluents et de mieux évaluer les coûts liés à leur traitement. On pourra intégrer cette modélisation dans le système d'aide à la décision pour le choix de solutions de valorisation de déchets du traitement de surface TS-Clean, décrit en première partie de cet article. L'objectif final de cet démarche est de mettre plus d'information à disposition des industriels pour leur permettre de choisir les solutions de traitement les mieux adaptées pour leurs déchets et effluents.

\footnotetext{
* Bruno Debray, Mathieu Leduc et Jacques Bourgois École des mines de Saint-Étienne - Département Ingénierie de l'environnement - 158, cours Fauriel - 42023 Saint-Étienne Cedex 2
}

Les notes de cet article se trouvent en bas de la page 52 